\title{
Cultural evidence for interpretation bias as a feature of social anxiety in Chinese adolescents
}

\author{
Meng Yu, P. Michiel Westenberg, Wei Li, Jianping Wang \& Anne C. Miers
}

To cite this article: Meng Yu, P. Michiel Westenberg, Wei Li, Jianping Wang \& Anne C. Miers (2019) Cultural evidence for interpretation bias as a feature of social anxiety in Chinese adolescents, Anxiety, Stress, \& Coping, 32:4, 376-386, DOI: 10.1080/10615806.2019.1598556

To link to this article: https://doi.org/10.1080/10615806.2019.1598556

曲 Published online: 29 Mar 2019.

Submit your article to this journal $\pi$

Џll Article views: 108

Q View related articles ¿

View Crossmark data \lceil 


\title{
Cultural evidence for interpretation bias as a feature of social anxiety in Chinese adolescents
}

\author{
Meng $\mathrm{Yu}^{\mathrm{a}, \mathrm{b}}$, P. Michiel Westenberg $\mathbb{B}^{\mathrm{b}}$, Wei $\mathrm{Li}^{\mathrm{c}}$, Jianping Wang ${ }^{\mathrm{a}}$ and Anne C. Miers ${ }^{\mathrm{b}}$ \\ ${ }^{a}$ Beijing Key Laboratory of Applied Experimental Psychology, National Demonstration Center for Experimental \\ Psychology Education, Faculty of Psychology, Beijing Normal University, Beijing, P.R. People's Republic of China; \\ bInstitute of Psychology, Developmental and Educational Psychology Unit, Leiden University, Leiden, Netherlands; \\ 'College of Educational Science and Technology, Northwest Minzu University, Lanzhou, P.R. People's Republic of \\ China
}

\begin{abstract}
Background and Objectives: Interpretation bias (IB), defined as the tendency to interpret ambiguous social situations in a threatening manner, has increasingly been studied in children and adolescents. Compared to Western samples, the relation between IB and social anxiety in Chinese youth has received little attention. The present study was to mainly examine the relationship between IB and social anxiety among Chinese adolescents.

Design: Cross-sectional design was utilized.

Methods: IB, measured by the Adolescents' Interpretation Bias Questionnaire (AIBQ), and social anxiety were surveyed among a group of high socially anxious Chinese adolescents $(n=25)$ and a control group $(n=29)$. Participants were asked to rate the likelihood of interpretations coming to mind in social/non-social situations and to choose the most believable interpretation.

Results: The high social anxiety group had more negative interpretations and beliefs in social situations, and the interpretation bias was particular to social anxiety versus depression. Additionally, the cognitive contentspecificity hypothesis was supported; the high anxious group showed interpretation bias in social situations, but didn't have more negative interpretations of non-social situations, after controlling for depression.

Conclusions: The present study yielded comparable findings as found in Western samples regarding the relation between IB and social anxiety.
\end{abstract}

\section{ARTICLE HISTORY}

Received 2 April 2018

Revised 18 October 2018

Accepted 22 January 2019

\section{KEYWORDS}

Chinese adolescents;

cognitive content specificity; cultural comparison; interpretation bias; social anxiety

\section{Introduction}

Social anxiety disorder (SAD) is defined by a fear of negative evaluation from others and of behaving in a way which is embarrassing or humiliating (DSM-5; American Psychiatric Association, 2013; Hirsch, Meeten, Krahé, \& Reeder, 2016). It is one of the most prevalent mental health disorders in youth populations (Kessler et al., 1994), with rates of about 10\% in studies with Western samples (Burstein et al., 2011; Merikangas et al., 2010). The presence of SAD during adolescence increases the risk of other comorbid disorders developing (Costello, Mustillo, Erkanli, Keeler, \& Angold, 2003). In addition, social anxiety symptoms or SAD in adolescence are associated with impairments in academic (e.g., Blöte, Miers, Heyne, \& Westenberg, 2015) and social functioning (e.g., Blöte \& Westenberg, 2007; La Greca \& Lopez, 1998). 
Relatively little is known about the prevalence of anxiety disorders and SAD specifically in nonWestern populations, such as China. National surveys of mental disorder prevalence in Chinese children and adolescents are not (yet) available. However, the available literature indicates a higher level of social anxiety among Chinese adolescents. For instance, among Chinese adolescents the average score on the Social Anxiety Scale for Adolescents (SAS-A; La Greca \& Lopez, 1998) is almost equal to North American adolescents' or above Spanish adolescents' clinical cut-off value (Zhou, Xu, Inglés, Hidalgo, \& La Greca, 2008) and also far higher than that of Dutch adolescents (Miers, Blöte, Bögels, \& Westenberg, 2008).

These above-mentioned findings suggest that the social anxiety level is highly prevalent among Chinese adolescents. However, little attention has been given to understanding the causal and maintenance factors related to social anxiety in this population. In the present study we focus specifically on interpretation bias, one of the key cognitive factors consistently related to social anxiety in Western samples (Stuijfzand, Creswell, Field, Pearcey, \& Dodd, 2017).

Several models have been proposed to explain how social anxiety develops. One of these is the Cognitive Model of Social Phobia developed by Clark and Wells (1995). According to this model, individuals with social anxiety have an inclination to interpret environmental cues, especially related to social or interpersonal scenarios, in a negative and catastrophic manner. Consequently, a negative interpretation bias is a key target in treatment such as Cognitive Behavioral Therapy (CBT). The tendency to negatively interpret ambiguous social situations is termed an "interpretation bias" (Clark \& Wells, 1995).

The current paper examines whether an interpretation bias is a characteristic of social anxiety in a non-Western culture, namely, China. Differences between cultures, with China being a collectivistic culture, whereas Western cultures are individualistic, could potentially impact the relationship between social anxiety and interpretation bias. Individuals in collectivistic cultures, compared to people in individualist cultures, are more likely to see themselves as a part of a group (Triandis, 2001). In addition, researchers (e.g., Benedict, 1946; Zhong et al., 2008) have noted that China, like other Eastern and collectivistic cultures has a "shame culture" society. Specifically, people are not encouraged to gaze at people, to be verbally assertive, to be explicit (Hofmann, Asnaani, \& Hinton, 2010). A potential consequence of these cultural features, collectivism and shame, is that people are less likely to interpret other people's social behavior negatively as this could offend or shame the group. Therefore, it could be possible that an interpretation bias is not as central to social anxiety, or might not be a typical characteristic of social anxiety as it is in Western cultures.

Research on interpretation bias and social anxiety in Western cultures originated from the adult population, demonstrating that individuals with a high level of social anxiety negatively interpret ambiguous social cues (e.g., Amir, Prouvost, \& Kuckertz, 2012; Constans, Penn, Ihen, \& Hope, 1999; Stopa \& Clark, 2000). Later, accumulating literature about interpretation bias and social anxiety in youth from Western countries yielded similar findings, with high socially anxious children and adolescents showing a negative bias in the interpretation of interpersonal-related information as compared to healthy controls or low anxious participants (e.g., Haller, Raeder, Scerif, Kadosh, \& Lau, 2016; Miers et al., 2008). Moreover, a recent meta-analysis of the association between negative interpretation and anxiety provides evidence for content specificity. That is, the association between negative interpretations and anxiety was larger when the anxiety subtype (e.g., social) matched the situation content (e.g., social) than when they did not match (Stuijfzand et al., 2017). These findings support the cognitive content-specificity hypothesis proposed by Beck (1979), indicating that each kind of emotional disturbance is correlated with a specific cognitive distortion. Although Stuijfzand et al. (2017) did not particularly investigate social anxiety, this review research still suggested a possibility of content-specificity in the relation between interpretation bias and social anxiety.

As well as focusing on negative interpretations, some researchers from Western countries (e.g., Giannini \& Loscalzo, 2016; Haller et al., 2016; Miers et al., 2008) have also explored the role of positive interpretations in the development and maintenance of social anxiety symptoms or clinical SAD 
among youth, however, demonstrating inconsistent findings. Some studies have found that high socially anxious youth demonstrate a paucity of positive interpretations. For instance, Haller et al. (2016) recruited 98 adolescents from the community to present them with ambiguous pictures in an experimental and computer-based manner. Results showed that adolescents with high social anxiety were more likely to interpret the situations as threatening, and came up with less positive interpretations towards ambiguous social situations. However, Miers et al. (2008) found that high socially anxious adolescents did not differ from their non-anxious peers on the extent to which positive interpretations of ambiguous social scenarios come to mind. Using the same hypothetical scenarios measure as Miers et al. (2008), Giannini and Loscalzo (2016) showed that Italian adolescents do have fewer positive interpretations of ambiguous social situations. Thus, in Western samples, the issue of whether socially anxious adolescents have fewer positive interpretations remains equivocal.

As well as examining negative and positive interpretations, a third area of interpretation bias research is related to Clark and Wells (1995) theory that social anxiety disorder arises from problematic beliefs about oneself and one's social world, which then leads individuals to interpret social situations in an excessively negative fashion (Hodson, McManus, Clark, \& Doll, 2008). A few studies have included a belief question in the measurement of interpretation bias (e.g., Miers et al., 2008; Stopa \& Clark, 2000). For example, in the Miers et al. (2008) study, 37 high socially anxious adolescents and 36 non-anxious adolescents were asked to report which interpretation (positive interpretation, negative interpretation or neutral interpretation) would be the most believable for them regarding each ambiguous scenario (social or non-social). Their results showed that, with regards to both social and non-social situations, high socially anxious adolescents tended to believe the threatening one. However, there was no significant effect for social anxiety group on negative belief in non-social situations after controlling for depression, which supported the cognitive content-specificity hypothesis (Miers et al., 2008). Similarly, Giannini and Loscalzo (2016) discovered that Italian adolescents high in social anxiety exhibit a tendency to show a greater belief in threatening interpretations towards social situations.

To the best of our knowledge, only one published and two unpublished studies have specifically investigated interpretation bias and social anxiety in Chinese adolescents. First, Yang (2011) showed, using a questionnaire to assess interpretation bias, that a socially anxious group reported more negative interpretations, with a lack of positive interpretations not found, in social situations. In contrast, using experimental IB paradigms, both greater negative interpretations and fewer positive interpretations towards social situations were shown in the high social anxiety group. A second study from Zhu (2016) demonstrated that the presence of threat interpretations on self-report questionnaires and an absence of positive interpretations via a computer-based paradigm positively predicted social anxiety level. Unfortunately, the latter study did not make a distinction between social and non-social situations and also did not control for depression, thus conclusions about content-specificity cannot be made. A third study focused on threat perception bias and anxiety among Chinese children and adolescents aged 9-19 years (Lu, Daleiden, \& Lu, 2007). Ten hypothetical audio-taped stories of ambiguous and non-ambiguous situations were used to assess threat interpretation bias. Anxiety was assessed in the different scenarios evoking generalized anxiety, social anxiety, and separation anxiety, respectively. The authors found that a higher level of anxiety in general was significantly correlated with threat perception, however, the content-specificity hypothesis was not supported, that is, youth more likely to perceive threat in social-anxiety-provoking vignettes were not inclined to report higher level of social anxiety (Lu et al., 2007). A possible explanation is that the study did not control for depressive symptoms. In sum, the existing evidence provides preliminary support for negatively biased interpretations among socially anxious Chinese adolescents, while the findings are equivocal for content-specificity and positive interpretations.

\subsection{The present study}

The aim of the current study was to investigate the relationship between interpretation bias and social anxiety in Chinese adolescents. To achieve this we conducted a replication of the Miers 
et al. (2008) study carried out in Dutch adolescents. The Miers et al. (2008) study used the Adolescents' Interpretation and Belief Questionnaire (AIBQ) to measure all interpretation bias components: negative and positive interpretations, and belief in both social and non-social situations. As depression is often shown to be comorbid with social anxiety (Essau, Conradt, \& Petermann, 1999; Ranta, Kaltiala-Heino, Rantanen, \& Marttunen, 2009), it is included as a covariate in the current study.

Based on the Miers et al. (2008) study, the following research questions were formulated; compared to a control group of non-anxious adolescents, do Chinese adolescents with a high level of social anxiety: (1) score significantly higher on negative interpretations but not lower on positive interpretations of social situations? (2) believe the negative interpretations of social situations? (3) show a tendency to negatively interpret ambiguous social situations even whilst controlling for depressive symptoms, demonstrating that the interpretation bias is particular to social anxiety versus depression? and (4) demonstrate an interpretation bias only in social situations, providing support for the cognitive content-specificity hypothesis?

A better understanding of whether an interpretation bias is a characteristic of high social anxiety in Chinese adolescents would inform clinical practice. Cognitive Behavioral Therapy (CBT) has been proved to be effective in targeting and treating social anxiety symptoms (disorder) in youth (e.g., Hayward et al., 2000). One of the essential therapeutic techniques is cognitive construction (e.g., McLellan, Alfano, \& Hudson, 2015), including targeting their interpretation bias (e.g., de Hullu, Sportel, Nauta, \& de Jong, 2017) and improving or augmenting positive thoughts (Vassilopoulos \& Brouzos, 2016), to make clients' cognitions less negatively biased. It is vital to gain knowledge about the role of positive and negative interpretations, and negative belief, in relation to social anxiety among Chinese adolescents before implementing CBT in this population.

\section{Methods}

\subsection{Participants}

A total of 251 adolescents, from one urban public school in Lanzhou, China, participated in this study. There were 107 boys and 155 girls, with a mean age of $16.69(S D=1.53)$ years and an average score of SAS-A of $50.47(S D=13.13)$. The parental marriage status of the majority of participants $(92.8 \%)$ was married, $4.0 \%$ was divorce, $0.8 \%$ was separated, and $0.8 \%$ reported widowed. According to the rule of the administrative division of China, $31.5 \%$ reported their place of residence was the countryside, $27.1 \%$ from the county/town, others (41.4\%) were from the city.

In order to make our results comparable, the social anxiety grouping method from Miers et al. (2008) was adopted. We checked for a gender difference on social anxiety and it was non-significant. Therefore, the same cut-off scores were used for boys and girls. According to the score on the measurement for social anxiety, the upper $10 \%$ adolescents was classified as the high social anxiety group, and participants scoring between the 45 and 55 percentile made up the control group. The high social anxiety group consisted of 25 participants (13 boys and 12 girls; $M \pm S D=$ $16.96 \pm 1.14$ years) and 29 adolescents made up the control group (12 boys and 17 girls; $M \pm S D=$ $16.45 \pm 3.31$ years $)$. There was a significant difference $[t(26$ adjusted d.f. $)=31.05, p<.001, d=9.09$ ] between the high social anxiety group $(M \pm S D=72.84 \pm 3.48)$ and the control group $(M \pm S D=$ $50.80 \pm .73$ ) on social anxiety. Participants in the two groups did not differ on gender, age, parental marriage status or place of residence.

\subsection{Measures}

Adolescents' Interpretation and Belief Questionnaire (AIBQ), developed by Miers et al. (2008), was used to measure adolescents' interpretations of five social and five non-social ambiguous situations. First, respondents were presented with each situation (social or non-social) followed by a specific question to address the ambiguity of the scenario, e.g., You have invited a group of classmates to your birthday 
party, but a few have not yet said if they are coming. Why haven't they said something yet? Then, three interpretations of the situation, positive, negative and neutral, were presented individually and respondents were asked to rate how likely each interpretation would appear in their mind (1-does not pop in my mind, 3-might pop in my mind, 5-definitely pops in my mind). Finally, respondents needed to choose which interpretation is the most believable. The Chinese version of AIBQ showed an acceptable convergent and discriminant validity, especially the social negative interpretation variable (Yu, Westenberg, Wang, Wang, \& Miers, 2019; submitted). In total, 6 subscales were used in the present study: negative interpretations, positive interpretations and negative belief (i.e., belief in negative interpretations) of both social and non-social situations. The standardized internal consistency coefficients (Eisinga, Grotenhuis, \& Pelzer, 2013) of negative interpretations and positive interpretations, respectively, in social and non-social situations, were .34 to .72. As the belief question asks the participants to make a choice between three interpretations and not to answer a question using a Likert scale, it is not suitable for computing Cronbach's alpha (Oliden \& Zumbo, 2008; Yu et al., 2019; submitted).

Social Anxiety Scale for Adolescents (SAS-A), developed by La Greca and Lopez (1998), contains 22 items: 18 items and four filler items. Each item, e.g., I worry that others don't like me, is rated on a 5point Likert scale according to how much the item "is true for you," ranging from 1 (not at all) to 5 (all the time). The Chinese version of SAS-A, including 18 descriptive items of social fears, was revised with a good reliability and validity (Zhou et al., 2008). The alpha coefficient of SAS-A in the present study was $.87(N=251)$.

Short Mood and Feelings Questionnaire (SMFQ; Angold, Costello, Pickles, \& Winder, 1995) was developed to measure depression in youth aged 8-16 years with ratings ranging from 0 (not true) to 2 (true). It consists of 13 items, for example, I feel no interest in everything, and showed good internal consistency ( $\alpha=88$ ) and test-retest reliability ( $\alpha=84$ ), respectively, in Chinese adolescents (Cheng, Cao, \& Su, 2009). In the present study, the internal consistency coefficient was .83 in a population of 251 adolescents.

\subsection{Procedure}

This study was approved by the Ethics Committee of Beijing Normal University. Once informed written consents were obtained from adolescents and parents, the participants received a package of measures, including demographic information, AIBQ, SAS-A and SMFQ. The questionnaires were collected anonymously; participants were in their classroom with a head teacher and a research experimenter to ensure the independent response from participants and make the process more clarified and smooth. After the completion of measurements, the experimenter or research assistant thanked the adolescents for their participation.

\subsection{Data analysis}

Participants whose missing data exceeded $20 \%$ or whose score on the SAS-A deviated by more than three standard deviations from the mean were excluded from the statistical analysis. This resulted in 29 participants being excluded and no participant was excluded for being an extreme case. The final sample included 251 participants. Little's MCAR chi-square test (Little \& Rubin, 2015) was not significant $(p=.29)$ suggesting that the data were missing at random. For cases with missing values less than $20 \%$ the missing values of SAS-A, AIBQ, and SFMQ were substituted with the mean item score in the sample. Standardized Cronbach coefficient was conducted with the four subscales of AIBQ because each subscale only has five items (Eisinga et al., 2013). MANOVAs were conducted with gender and social anxiety group as independent variables and social and non-social negative interpretations, positive interpretations and negative belief, as dependent variables, followed up with univariate ANOVAs. To examine the cognitive content-specificity hypothesis, additional MANCOVAs were conducted with the score on SMFQ for depression as the control variable. Analyses were 
conducted in SPSS 22.0. Due to its powerfulness and robustness to the homogeneity hypothesis, Pillai's Trace statistic is reported in all MANOVA and MANCOVA results (Tabachnick \& Fidell, 2007).

\section{Results}

\subsection{Interpretations and belief in social situations}

To examine the first and second research questions, a 2 (social anxiety group) x 2 (gender) MANOVA was conducted with positive and negative interpretations and belief of interpretations for social situations as the dependent variables (Table 1 displays the means and standard deviations of the AIBQ variables in social situations by social anxiety group). The multivariate test yielded a significant effect for social anxiety group $\left[F(3,48)=4.71, p=.006\right.$, partial $\left.\eta^{2}=.23\right]$. A main effect of gender and the interaction effect of social anxiety group by gender were non-significant. Subsequent ANOVAs demonstrated that, compared to the control group, adolescents with high level of social anxiety were characterized by higher scores on negative interpretations $\left[F(1,50)=14.09, p<.001, \eta_{p}^{2}=.22\right]$ and negative belief $\left[F(1,50)=7.19, p=.01, \eta_{p}^{2}=.13\right]$, but did not differ on positive interpretations.

\subsection{Interpretation bias: specific to social anxiety?}

To examine the third research issue of the present study, a second MANOVA, similar to the first, was conducted with the addition of the SMFQ score as a covariate to control for the influence of depression symptoms. The multivariate tests showed a significant effect of SMFQ on adolescents' interpretations and belief, $F(3,47)=4.53, p=.007, \eta_{p}^{2}=.22$, however, demonstrating a significant effect of depression only on positive interpretations, $F(1,49)=9.33, p=.004, \eta_{p}^{2}=.16$. After controlling for depressive emotion, social anxiety group still had a significant main effect $[F(3,47)=3.24, p=.03$, $\left.\eta_{p}^{2}=.17\right]$. The between-subjects effects displayed a significant social anxiety group effect for negative interpretations $\left[F(1,49)=5.94, p=.02, \eta_{p}^{2}=.11\right]$, positive interpretations $[F(1,49)=6.29, p=.02$, $\left.\eta_{p}^{2}=.11\right]$ and belief $\left[F(1,49)=4.49, p=.04, \eta_{p}^{2}=.08\right]$, after partialling out the effect of depression.

\subsection{Cognitive content-specificity of interpretation bias}

To test the fourth research issue, an additional 2 (social anxiety group) $\times 2$ (gender) MANOVA was conducted with non-social negative and positive interpretations, and negative belief as dependent variables (Table 2 shows the means and standard deviations from this analysis by social anxiety). Only the multivariate effect of social anxiety group $\left[F(3,48)=4.90, p=.005, \eta_{p}^{2}=.23\right]$ was significant. Neither the main effect of gender nor the interaction effect of gender and social anxiety group showed was significant. Follow-up ANOVAs demonstrated that the high social anxiety group reported higher scores on negative interpretations $\left[F(1,50)=13.85, p=.001, \eta_{p}^{2}=.22\right]$, and was more likely to believe these negative interpretations $\left[F(1,50)=7.67, p=.008, \eta_{p}^{2}=.13\right]$. There was no significant difference between high social anxiety group and control group on non-social positive interpretations.

To figure out whether the significant social anxiety group effect could be accounted for by depression symptoms, a follow-up MANCOVA was conducted with depression as covariate. The

Table 1. AIBQ social situations: negative and positive interpretations and negative belief.

\begin{tabular}{lllll}
\hline & Social anxiety group & $M$ & $S D$ & $n$ \\
\hline Negative interpretations & Control & 2.55 & .74 & .91 \\
Positive interpretations & High SA & 3.33 & .71 & 29 \\
& Control & 2.48 & .62 & 25 \\
Negative belief & High SA & 2.35 & .29 & 25 \\
& Control & 2.03 & .42 & 29 \\
\hline
\end{tabular}

Note. $\mathrm{AIBQ}=$ Adolescents' Interpretation and Belief Questionnaire; SA = Social anxiety. 
Table 2. AIBQ non-social situations: negative and positive interpretations and belief in negative interpretations.

\begin{tabular}{lllll}
\hline & Social anxiety group & $M$ & $S D$ & $n$ \\
\hline Negative interpretation & Control & 2.58 & .70 & 29 \\
& High SA & 3.22 & .64 & .72 \\
Positive interpretation & Control & 3.64 & .70 & 25 \\
Negative belief & High SA & 3.45 & .39 & 29 \\
& Control & 1.53 & .35 & 29 \\
\hline
\end{tabular}

Note: $\mathrm{AIBQ}=$ Adolescents' Interpretation and Belief Questionnaire; SA = Social anxiety.

multivariate tests did not yield a significant main effect for depression, $F(3,47)=2.55, p=.07, \eta_{p}^{2}=.14$. After partialling out the effect of depression, the findings no longer showed a significant social anxiety group effect in non-social situations.

Combined with the findings from sections 3.1 and 3.2, social anxiety group showed a significant effect on negative interpretations and belief in social situations even after controlling for depression whereas there was no significant effect in non-social situations, providing supportive evidence for cognitive content-specificity.

\section{Discussion}

Biases in the interpretation of ambiguous social scenarios are central to cognitive models of social anxiety (disorder). To culturally examine the relationship between interpretation bias and social anxiety in Chinese adolescents, the present study replicated the Miers et al. (2008) study in Dutch adolescents. Overall, the current study contributed a first effort to simultaneously focus on all components of interpretation bias, and our findings supported the cognitive content-specificity hypothesis in a Chinese adolescent sample.

In line with Miers et al. (2008) and a study in a Chinese population (Yang, 2011), socially anxious participants reported a higher level of negative interpretations and were not significantly associated with lower positive interpretations in social situations. The findings also showed that high social anxiety participants were more likely to believe the negative interpretations when confronting the ambiguous social situations. This finding provides preliminarily support for Clark and Wells (1995) model of social phobia from the perspective of a different, collectivist culture. Moreover, consistent with Miers et al. (2008) and other studies in Western adolescents (e.g., Italy; Giannini \& Loscalzo, 2016), Chinese adolescents' social interpretation bias was particular to social anxiety symptoms and not reflective of an underlying factor common to different internalizing disorders, as shown by the finding that interpretation bias remained significant after controlling for depressive symptoms. Finally, the findings in the current study were in support of the cognitive content-specificity hypothesis, indicating that the high socially anxious group significantly endorsed negative interpretations only in social (and not non-social) situations, after controlling for depression. In sum, the current study's findings suggest that the relationship between interpretation bias and social anxiety as found in Western samples could be extended to a non-Western, Chinese culture.

Interestingly, the effect of depression on positive interpretations in social situations was not in line with Miers et al. (2008). The present findings showed a social anxiety group effect for positive interpretations after controlling for depressive symptoms. That is, positive interpretations were significantly less likely to come to mind in socially anxious adolescents compared with the control group, when holding the depression level equal in the two groups. This is in line with the findings from Giannini and Loscalzo (2016) in Italian adolescents. Taken together these findings seem to imply that in Italian and Chinese socially anxious adolescents, a positive bias is lacking whereas a positive bias is present in Dutch adolescents with high levels of social anxiety. Moreover, previous studies have found that, when conducting cognitive behavioral therapy (CBT) for youth with anxiety disorders, it was the increase of positive interpretations, not the change in negative interpretations, that preceded a decrease of post-treatment anxious symptoms (e.g., Hogendoorn et al., 2014). 
Therefore, although this finding was unexpected based on the Miers et al. (2008) study, combined with other empirical evidence it provides an indication that positive interpretations might be an important target for social anxiety interventions in Western and non-Western populations.

Existing literature has suggested that the level of social anxiety among Chinese adolescents is higher than that in Western samples (e.g., Zhou et al., 2008). Our findings support this; Chinese adolescents exhibited a much higher mean score of social anxiety compared to that in Miers et al. (2008) (e.g., normal group, 50.47 v.s. 34.67 ; high social anxiety group, 72.84 v.s. 55.46). Interestingly, despite the presence of higher social anxiety levels, the mean score of negative interpretations and belief in social situations in each group was similar to that from the study of Miers et al. (2008). Thus, the high anxious Chinese adolescents scored higher on the SAS-A compared to the Dutch adolescents, but the interpretation bias scores were practically equivalent in the two cultures. SAS-A has been culturally validated in China (Zhou et al., 2008), nevertheless, a clinical cut-off was not provided. It seems that the clinical cut-off value of SAS-A, originated from Western populations, would not apply to Chinese adolescents, making it incomparable among different cultural backgrounds. Establishing a clinical cut-off on the SAS-A among Chinese population is highly recommended in future research.

Although the present study yielded important results on the relationship between interpretation bias and social anxiety among Chinese socially anxious adolescents, it was still only a preliminary investigation. Some limitations need to be considered. First, the small sample size should be noted and the results can only be generalized to rural adolescents or community samples with caution. As we did not include a group of adolescents with a SAD diagnosis we cannot generalize to clinical patients. Second, only self-reported data about symptoms and cognitions were collected in the present study, other resources, for instance, parent-reported data and clinician evaluation, would be considered in future research for acquiring more complete information. To further examine the characteristics of interpretation bias in the maintenance of social anxiety, for instance, its stability over time, a follow-up study would be required. Third, we acknowledge the low reliabilities of AIBQ subscales (except negative social). In a psychometric paper about the AIBQ in Chinese adolescents, they are higher with a larger sample size (Yu et al., 2019, submitted). Finally, as Halldorsson and Creswell (2017) suggested, the specificity between interpretation bias and social anxiety, not other type of anxiety disorders, needs to be further investigated.

In conclusion, the findings support the presence of an interpretation bias in socially anxious Chinese adolescents. In the cognitive model of social phobia proposed by Clark and Wells (1995), interpretation bias is a key factor involved in maintenance of social anxiety symptoms/disorder. Therefore, future research could focus on interpretation bias to help lessen social anxiety symptoms in Chinese adolescents. For instance, some researchers have developed an Interpretation Bias Modification (IBM) technique to intervene in early emerging social anxiety symptoms during adolescence and this has been shown to be effective (e.g., Lothmann, Holmes, Chan, \& Lau, 2011). Moreover, group CBT has been evidenced to effectively improve anxiety symptoms in Chinese youth (e.g., Sun, Liu, \& Cheng, 2014; Yan, Wang, Tang, Wang, \& Xie, 2015). We suggest that, by adjusting the way of thinking, such as, the negative interpretation "They don't like me," socially anxious Chinese adolescents could benefit from IBM.

In addition, the current study adds to a small body of research that is suggestive of a higher level of social anxiety among Chinese adolescents than that in Western samples. However, the finding with respect to the practically similar score on the measure of negative interpretations in socially anxious Chinese and Dutch adolescents may indicate that other factors, such as culture-related factors, would increase the severity of social anxiety symptoms among Chinese adolescents. For example, cultural language analysis about the attitude towards anxiety between Chinese (the East) and English (the West), found that, except for the common components (e.g., restlessness, worry), English also includes the optimistic component, desire (Liu, 2007). In the meta-cognitive model of generalized anxiety disorder, Wells (2005) postulated that the meta-worry (i.e., worry about worry) would further stir up anxiety. Therefore, a possible explanation that a less optimistic attitude towards anxiety, similar to meta-worry, in Chinese, to some extent would increase anxiety. Thus differences 
in anxiety level, including social anxiety, between the East and West, may partially be due to the cultural background and its language. Moreover, findings from Jose, Kramar, and Hou (2014) research in Chinese and New Zealand adolescents showed that reporting a higher level of rumination in Chinese adolescents may be due to their stronger collectivist orientation in interpersonal relationships. This suggests that culture-related cognitive differences, such as, rumination, could exacerbate the maintenance of anxiety symptoms, including social anxiety. We suggest that future research pay more attention to these culturally significant cognitive factors in Chinese adolescents and their relation to social anxiety.

\section{Acknowledgements}

The authors would like to thank Professor Linyan Su for providing the helpful information, clinically and academically, about Chinese children and adolescents' anxiety.

\section{Disclosure statement}

No potential conflict of interest was reported by the authors.

\section{Funding}

The current research is funded by the China Scholarship Council.

\section{ORCID}

P. Michiel Westenberg (D) http://orcid.org/0000-0001-9138-7703

\section{References}

American Psychiatric Association. (2013). Diagnostic and statistical manual of mental disorders (5th ed.). Washington, DC: American Psychiatric Association.

Amir, N., Prouvost, C., \& Kuckertz, J. M. (2012). Lack of a benign interpretation bias in social anxiety disorder. Cognitive Behaviour Therapy, 41(2), 119-129. doi:10.1080/16506073.2012.662655

Angold, A., Costello, E. J., Pickles, E. J., \& Winder, F. (1995). The development of a questionnaire for use in epidemiological studies of depression in children and adolescents. International Journal of Methods in Psychiatric Research, 5(4), 237249. doi:1049-8931.95/040237-13

Beck, A. T. (1979). Cognitive therapy and the emotional disorders. New York: Penguin.

Benedict, R. (1946). The chrysanthemum and the sword: Patterns of Japanese culture. Boston: Houghton Mifflin.

Blöte, A. W., Miers, A. C., Heyne, D. A., \& Westenberg, P. M. (2015). Social anxiety and the school environment of adolescents. In Social anxiety and phobia in adolescents. Springer International Publishing. doi:10.1007/978-3-319-16703-9

Blöte, A. W., \& Westenberg, P. M. (2007). Socially anxious adolescents' perception of treatment by classmates. Behaviour Research and Therapy, 45(2), 189-198. doi:10.1016/j.brat.2006.02.002

Burstein, M., He, J. P., Kattan, G., Albano, A. M., Avenevoli, S., \& Merikangas, K. R. (2011). Social phobia and subtypes in the national comorbidity survey-adolescent supplement: prevalence, correlates, and comorbidity. Journal of the American Academy of Child and Adolescent Psychiatry, 50(9), 870-880. doi:10.1016/j.jaac.2011.06.005

Cheng, P., Cao, F., \& Su, L. (2009). Reliability and validity of the short mood and feelings questionnaire in Chinese adolescents. Chinese Mental Health Journal, 23(1), 60-63. doi:10.3969/j.issn.1000-6729.2009.01.016

Clark, D. M., \& Wells, A. (1995). A cognitive model of social phobia. In R. G. Heimberg, M. R. Liebowitz, D. A. Hope, \& F. R. Schneier (Eds.), Social phobia: diagnosis, assessment, and treatment (pp. 69-93). New York: The Guildford Press.

Constans, J. I., Penn, D. L., Ihen, G. H., \& Hope, D. A. (1999). Interpretive biases for ambiguous stimuli in social anxiety. Behaviour Research and Therapy, 37(7), 643-651. doi:10.1016/S0005-7967(98)00180-6

Costello, E. J., Mustillo, S., Erkanli, A., Keeler, G., \& Angold, A. (2003). Prevalence and development of psychiatric disorders in childhood and adolescence. Archives of General Psychiatry, 60(8), 837-844. doi:10.1001/archpsyc.60.8.837

de Hullu, E., Sportel, B. E., Nauta, M. H., \& de Jong, P. J. (2017). Cognitive bias modification and CBT as early interventions for adolescent social and test anxiety: Two-year follow-up of a randomized controlled trial. Journal of Behavior Therapy and Experimental Psychiatry, 55, 81-89. doi:10.1016/j.jbtep.2016.11.011 
Eisinga, R., Grotenhuis, M. T., \& Pelzer, B. (2013). The reliability of a two-item scale: Pearson, Cronbach, or SpearmanBrown? International Journal of Public Health, 58(4), 637-642. doi:10.1007/s00038-012-0416-3

Essau, C. A., Conradt, J., \& Petermann, F. (1999). Frequency and comorbidity of social phobia and social fears in adolescents. Behaviour Research and Therapy, 37(9), 831-843.

Giannini, M., \& Loscalzo, Y. (2016). Social anxiety and adolescence: Interpretation bias in an Italian sample. Scandinavian Journal of Psychology, 57(1), 65-72. doi:10.1111/sjop.12263

Halldorsson, B., \& Creswell, C. (2017). Social anxiety in pre-adolescent children: What do we know about maintenance? Behaviour Research and Therapy, 99, 19-36. doi:10.1016/j.brat.2017.08.013

Haller, S. P. W., Raeder, S. M., Scerif, G., Kadosh, K. C., \& Lau, J. Y. F. (2016). Measuring online interpretations and attributions of social situations: Links with adolescent social anxiety. Journal of Behavior Therapy and Experimental Psychiatry, 50, 250-256. doi:10.1016/j.jbtep.2015.09.009

Hayward, C., Varady, S., Albano, A. M., Thienemann, M., Henderson, L., \& Schatzberg, A. F. (2000). Cognitive-Behavioral group therapy for social phobia in female adolescents: Results of a pilot study. Journal of the American Academy of Child \& Adolescent Psychiatry, 39(6), 721-726. doi:10.1097/00004583-200006000-00010

Hirsch, C. R., Meeten, F., Krahé, C., \& Reeder, C. (2016). Resolving ambiguity in emotional disorders: The nature and role of interpretation biases. Annual Review of Clinical Psychology, 12(1), 281-305. doi:10.1146/annurev-clinpsy-021815093436

Hodson, K. J., McManus, F. V., Clark, D. M., \& Doll, H. (2008). Can clark and wells' (1995) cognitive model of social phobia be applied to young people? Behavioural and Cognitive Psychotherapy, 36(4), 449-461. doi:10.1017/S1352465808004487

Hofmann, S. G., Asnaani, A., \& Hinton, D. E. (2010). Cultural aspects in social anxiety and social anxiety disorder. Depression and Anxiety, 27(12), 1117-1127. doi:10.1002/da.20759

Hogendoorn, S. M., Prins, P. J. M., Boer, F., Vervoort, L., Wolters, L. H., Moorlag, H., ... de Haan, E. (2014). Mediators of cognitive behavioral therapy for anxiety-disordered children and adolescents: Cognition, perceived control, and coping. Journal of Clinical Child and Adolescent Psychology, 43(3), 486-500. doi:10.1080/15374416.2013.807736

Jose, P. E., Kramar, K., \& Hou, Y. (2014). Does brooding rumination moderate the stress to depression relationship similarly for Chinese and New Zealand adolescents? Journal of Educational and Developmental Psychology, 4(1), $114-127$. doi:10.5539/jedp.v4n1p114

Kessler, R. C., Mcgonagle, K. A., Zhao, S., Nelson, C. B., Hughes, M., Eshleman, S., ... Kendler, K. S. (1994). Lifetime and 12Month prevalence of DSM-III-R psychiatric disorders in the United States. Archives of General Psychiatry, 51(1), 8-19. doi:10.1001/archpsyc.1994.03950010008002

La Greca, A. M., \& Lopez, N. (1998). Social anxiety amon adolescents : Linkages with peer relation s and friendships. Journal of Abnormal Child Psychology, 26(2), 83-94. doi:10.1023/A:1022684520514

Little, R. J., \& Rubin, D. B. (2015). Missing data. International Encyclopedia of the Social \& Behavioral Sciences, 15, $602-607$. doi:10.1016/B978-0-08-097086-8.42082-9

Liu, X. (2007). Settles on western from the Chinese-English two languages anxiousview. Journal of Hubei TV University, 27 (9), 104-105. doi:1008-7427(2007)09-0104-02

Lothmann, C., Holmes, E. A., Chan, S. W., \& Lau, J. Y. F. (2011). Cognitive bias modification training in adolescents: Effects on interpretation biases and mood. Journal of Child Psychology and Psychiatry, 52(1), 24-32. doi:10.1111/j.1469-7610. 2010.02286.x

Lu, W., Daleiden, E., \& Lu, S. (2007). Threat perception bias and anxiety among Chinese school children and adolescents. Journal of Clinical Child \& Adolescent Psychology, 36(4), 568-580. doi:10.1080/15374410701776301

McLellan, L. F., Alfano, C. A., \& Hudson, J. L. (2015). Cognition-focused interventions for social anxiety disorder among adolescents. In K. Ranta, A. M. La Greca, L.-J. Garcia-Lopez, \& M. Marttunen (Eds.), Social anxiety and phobia in adolescents: Development, manifestation and intervention strategies (pp. 225-250). Switzerland: Springer International Publishing. doi:10.1007/978-3-319-16703-9

Merikangas, K. R., He, J., Burstein, M., Swanson, S. A., Avenevole, S., Cui, L., ... Swendsen, J. (2010). Lifetime prevalence of mental disorders in U.S. adolescents: Results from the National Comorbidity study - adolescent supplement (NCS-A). Journal of American Accad Child Adolescen Psychiatry, 49(10), 980-989.

Miers, A. C., Blöte, A. W., Bögels, S. M., \& Westenberg, P. M. (2008). Interpretation bias and social anxiety in adolescents. Journal of Anxiety Disorders, 22(8), 1462-1471. doi:10.1016/j.janxdis.2008.02.010

Oliden, P. E., \& Zumbo, B. D. (2008). Reliability coefficients for ordinal response scales. Psicothema, 20(4), 896-901. doi: ISSN:0214-9915

Ranta, K., Kaltiala-Heino, R., Rantanen, P., \& Marttunen, M. (2009). Social phobia in Finnish general adolescent population: Prevalence, comorbidity, individual and family correlates, and service use. Depression and Anxiety, 26(6), 528-536. doi:10.1002/da.20422

Stopa, L., \& Clark, D. M. (2000). Social phobia and interpretation of social events. Behaviour Research and Therapy, 38(3), 273-283. doi:10.1016/S0005-7967(99)00043-1

Stuijfzand, S., Creswell, C., Field, A. P., Pearcey, S., \& Dodd, H. (2017). Research review: Is anxiety associated with negative interpretations of ambiguity in children and adolescents? A systematic review and meta-analysis. Journal of Child Psychology and Psychiatry. doi:10.1111/jcpp.12822 
Sun, Y., Liu, W., \& Cheng, W. (2014). 3-month follow-up study for group cognitive behavioral treatment on adolescents with anxiety disorders. Shanghai Journal of Clinical Psychiatry, 24(2), 94-96. doi:1005-3220(2014)02-0094-03

Tabachnick, B. G., \& Fidell, L. S. (2007). Using multivariate statistics (5th ed.). Boston: Allyn \& Bacon/Person Education.

Triandis, H. C. (2001). Individualism-collectivism and personality. Journal of Personality, 69(6), 907-924.

Vassilopoulos, S. P., \& Brouzos, A. (2016). Cognitive bias Modification of interpretations in children: Processing information about ambiguous social events in a Duo. Journal of Child and Family Studies, 25(1), 299-307. doi:10.1007/s10826-0150194-7

Wells, A. (2005). The metacognitive model of GAD: Assessment of meta-worry and relationship with DSM-IV generalized anxiety disorder. Cognitive Therapy and Research, 29(1), 107-121. doi:10.1007/s10608-005-1652-0

Yan, Y. L., Wang, S. S., Tang, M., Wang, J. P., \& Xie, Q. Y. (2015). Effects of group cognitive-behavioural therapy on adolescent anxiety symptoms. Chinese Mental Health Journal, 29(1), 10-15. doi:10.3969/j.issn.1000-6729.2015.01.003

Yang, J. (2011). Interpretation bias in adolescents with socian anxiety (Master's Thesis). Southwest University.

Yu, M., Westenberg, P. M., Wang, Y., Wang, J., \& Miers, A. C. (2019). Psychometric properties of the Adolescents' Interpretation and Belief Questionnaire (AIBQ) in Chinese adolescents. Submitted manuscript, Faculty of Psychology, Beijing Normal University, Beijing, China.

Zhong, J., Wang, ÃA, Qian, M., Zhang, L., Gao, J., Yang, J., ... Chen, P. (2008). Shame, personality, and social anxiety symptoms in Chinese and American nonclinical samples: A cross-cultural study. Depression and Anxiety, 25(5), 449-460. doi:10.1002/da.20358

Zhou, X., Xu, Q., Inglés, C. J., Hidalgo, M. D., \& La Greca, A. M. (2008). Reliability and validity of the Chinese version of the social anxiety scale for adolescents. Child Pyschiatry \& Human Development, 39(2), 185-200. doi:10.1007/s10578-0070079-0

Zhu, S. (2016). The relatinships among parental Style and interpretation bias on social anxiety in Child aged 11-15 (Master's Thesis). Zhejiang Sci-Tech University. 